\title{
CONTINUED FRACTIONS AND CLASS NUMBER TWO
}

\author{
RICHARD A. MOLLIN
}

(Received 12 September 2000)

\begin{abstract}
We use the theory of continued fractions in conjunction with ideal theory (often called the infrastructure) in real quadratic fields to give new class number 2 criteria and link this to a canonical norm-induced quadratic polynomial. By doing so, this provides a real quadratic field analogue of the well-known result by Hendy (1974) for complex quadratic fields. We illustrate with several examples.
\end{abstract}

2000 Mathematics Subject Classification. 11R11, 11A55, 11R29.

1. Introduction. In [4], we gave criteria for real quadratic fields of ERD-type (those with discriminant of the form $\Delta=\ell^{2}+r$ with $r \mid 4 \ell$ ) to have class number 2 in terms of a canonical quadratic polynomial, thus providing a real quadratic field analogue of a result of Hendy [2] for complex quadratic fields. Herein we give more general criteria for (not necessarily ERD type) real quadratic fields in terms of the same polynomial using the theory of the infrastructure. Moreover, we are able to handle certain ERD types that were not covered in [4].

2. Notation and preliminaries. In this section, we see how ideal theory is linked to continued fractions via what the late Dan Shanks (see [6]) called the infrastructure of a real quadratic field.

First we need the following notion.

Definition 2.1. Suppose that $D \in \mathbb{N}$ is not a perfect square. Then a quadratic irrational is a number of the form

$$
\alpha=\frac{P+\sqrt{D}}{Q}, \quad(P, Q \in \mathbb{Z}),
$$

where $Q \neq 0$ and $P^{2} \equiv D(\bmod Q)$. Also, the algebraic conjugate of $\alpha$ is

$$
\alpha^{\prime}=\frac{P-\sqrt{D}}{Q} .
$$

We are interested in linking this notion to both continued fractions and ideals. To establish the link with continued fractions, we first note that it is well known that a real number has a periodic continued fraction expansion if and only if it is a quadratic irrational (see [7, Theorem 5.3.1, page 240]). Furthermore a quadratic irrational may have a purely periodic continued fraction expansion which we denote by

$$
\alpha=\left\langle\overline{q_{0} ; q_{1}, q_{2}, \ldots, q_{\ell-1}}\right\rangle,
$$


meaning that $q_{n}=q_{n+\ell}$ for all $n \geq 0$, where $\ell=\ell(\alpha)$ is the period length of the simple continued fraction expansion. It is known that a quadratic irrational $\alpha$ has such a purely periodic expansion if and only if $\alpha>1$ and $-1<\alpha^{\prime}<0$. Any quadratic irrational which satisfies these two conditions is called reduced (see [7, Theorem 5.3.2, page 241]). Now we are in a position to bring in the theory of ideals and link the three together.

Let $D>1$ be a squarefree positive integer and set

$$
\sigma= \begin{cases}2 & \text { if } D \equiv 1(\bmod 4) \\ 1 & \text { otherwise }\end{cases}
$$

Define

$$
\omega_{\Delta}=\frac{\sigma-1+\sqrt{D}}{\sigma}, \quad \Delta=\left(\omega_{\Delta}-\omega_{\Delta}^{\prime}\right)^{2}=\frac{4 D}{\sigma^{2}} .
$$

The value $\Delta$ is called a (fundamental) discriminant or (field) discriminant with associated radicand $D$, and $\omega_{\Delta}$ is called the (fundamental) principal surd associated with $\Delta$. For simplicity, we will refer to $\Delta$ as a discriminant with associated radicand $D$ and principal surd $\omega_{\Delta}$. This will provide the canonical basis element for certain rings that we now define.

Let $[\alpha, \beta]=\alpha \mathbb{Z}+\beta \mathbb{Z}$ be a $\mathbb{Z}$-module. Then

$$
\wp_{\Delta}=\left[1, \omega_{\Delta}\right]
$$

is the ring of integers of $K=\mathbb{Q}(\sqrt{\Delta})=\mathbb{Q}(\sqrt{D})$. The fundamental unit of $\bigodot_{\Delta}$ is denoted by $\varepsilon_{\Delta}$.

It may be shown that any $\mathbb{Z}$-module $I \neq(0)$ of $\oplus_{\Delta}$ has a representation of the form $\left[a, b+c \omega_{\Delta}\right]$, where $a, c \in \mathbb{N}$ with $0 \leq b<a$. We will only be concerned with primitive $\mathbb{Z}$-modules, namely those for which $c=1$. In other words, $I$ is a primitive $\mathbb{Z}$-submodule of $\Theta_{\Delta}$ if whenever $I=(z) J$ for some $z \in \mathbb{Z}$ and some $\mathbb{Z}$-submodule $J$ of $\oplus_{\Delta}$, then $|z|=1$. Thus, a canonical representation of a primitive $\mathbb{Z}$-submodule of $\wp_{\Delta}$ is obtained by setting $\sigma a=Q$ and $b=(P-1) / 2$ if $\sigma=2$, while $b=P$ if $\sigma=1$ for $P, Q \in \mathbb{Z}$, namely

$$
I=\left[\frac{Q}{\sigma}, \frac{P+\sqrt{D}}{\sigma}\right]
$$

Now we set the stage for linking ideal theory with continued fractions by giving a criterion for a primitive $\mathbb{Z}$-module to be a primitive ideal in $\oplus_{\Delta}$. A nonzero $\mathbb{Z}$-module $I$ as given in (2.7) is called a primitive $\bullet_{\Delta}$-ideal if and only if $P^{2} \equiv D(\bmod Q)$ (see [7, Theorem 3.5.1, page 173]). Henceforth, when we refer to an $\wp_{\Delta}$-ideal it will be understood that we mean a primitive $\odot_{\Delta}$-ideal. Also, the value $Q / \sigma$ is called the norm of $I$, denoted by $N(I)$. Hence, we see that $I$ is an $\bullet_{\Delta}$-ideal if and only if $\alpha=(P+\sqrt{D}) / Q$ is a quadratic irrational. Given the notion of a reduced quadratic irrational discussed earlier, it is not surprising that we define a reduced ideal $I$ to be one which contains an element $\beta=(P+\sqrt{D}) / \sigma$ such that $I=[N(I), \beta]$, where $\beta>N(I)$ and $-N(I)<\beta^{\prime}<0$, since this corresponds exactly to the reduced quadratic irrational $\alpha=\beta / N(I)>1$ with $-1<\alpha^{\prime}<0$. In fact, the following theorem holds. 
THEOREM 2.2. Let $\Delta$ be a discriminant with associated radicand $D$. Then $I=[Q / \sigma$, $(P+\sqrt{D}) / \sigma]$ is a reduced $\oplus_{\Delta}$-ideal if $Q / \sigma<\sqrt{\Delta} / 2$. Conversely, if $I$ is a reduced $\wp_{\Delta}$-ideal, then $Q / \sigma<\sqrt{\Delta}$.

Proof. See [7, Corollaries 5.5.1 and 5.5.2, page 259].

Also, it is not surprising that we define the conjugate ideal of $I$ to be

$$
I^{\prime}=\left[\frac{Q}{\sigma}, \frac{P-\sqrt{D}}{\sigma}\right]
$$

Now we link continued fractions to the ideals defined above. Let $I$ be an $\oplus_{\Delta}$-ideal given by (2.7). Define

$$
\begin{gathered}
P_{0}=P, \quad Q_{0}=Q, \quad \text { and recursively for } j \geq 0, \\
q_{j}=\left\lfloor\frac{P_{j}+\sqrt{D}}{Q_{j}}\right\rfloor, \quad P_{j+1}=q_{j} Q_{j}-P_{j}, \quad D=P_{j+1}^{2}+Q_{j} Q_{j+1} .
\end{gathered}
$$

It follows that we have the simple continued fraction expansion of $\alpha$ given by

$$
\alpha=\frac{P+\sqrt{D}}{Q}=\frac{P_{0}+\sqrt{D}}{Q_{0}}=\left\langle q_{0} ; q_{1}, \ldots, q_{j}, \ldots\right\rangle
$$

Now the stage is set for the appearance of the result that formally merges ideals

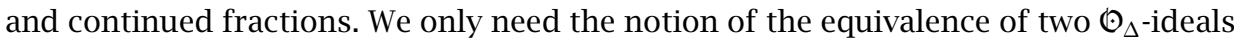
$I$ and $J$, denoted $I \sim J$ to proceed. We write $I \sim J$ to denote the fact that there exist nonzero integers $\alpha, \beta \in \mathcal{\odot}_{\Delta}$ such that $(\alpha) I=(\beta) J$, where $(x)$ denotes the principal $\wp_{\Delta}$-ideal generated by $x \in \odot_{\Delta}$. For a given discriminant $\Delta$, the class group of $\odot_{\Delta}$ determined by these equivalence classes, denoted by $\mathfrak{C}_{\Delta}$, is of finite order, denoted by $h_{\Delta}$, called the class number of $\oplus_{\Delta}$. One result on the class group that we will need in the next section is the following.

THEOREM 2.3. If $\Delta$ is a discriminant, then every class of $\mathfrak{C}_{\Delta}$ contains a primitive ideal $I$ with $N(I) \leq \sqrt{\Delta} / 2$. Furthermore, $\mathscr{C}_{\Delta}$ is generated by the primitive non-inert prime ideals $\mathscr{P}$ with $N(\mathscr{P})<\sqrt{\Delta} / 2$

Proof. See [5, Theorem 1.3.1, page 15].

Now we may present the continued fraction algorithm.

THEOREM 2.4. Suppose that $\Delta \in \mathbb{N}$ is a discriminant, $P_{j}, Q_{j}$ are given by (2.10) and

$$
I_{j}=\left[\frac{Q_{j-1}}{\sigma}, \frac{P_{j-1}+\sqrt{D}}{\sigma}\right]
$$

for nonnegative $j \in \mathbb{Z}$. Then $I_{1} \sim I_{j}$ for all $j \in \mathbb{N}$. Furthermore, there exists a least natural number $n$ such that $I_{n+j}$ is reduced for all $j \geq 0$, and these $I_{n+j}$ are all of the reduced ideals equivalent to $I_{1}$. If $\ell \in \mathbb{N}$ is the least value such that $I_{n}=I_{\ell+n}$, then for $j \geq n-1, \alpha_{j}=\left(P_{j}+\sqrt{D}\right) / Q_{j}$ all have the same period length $\ell=\ell\left(\alpha_{j}\right)=\ell\left(\alpha_{n-1}\right)$. 
Proof. See [7, Theorem 5.5.2, pages 261-266].

From the continued fraction algorithm, we see that if

$$
I=\left[\frac{Q}{\sigma}, \frac{P+\sqrt{D}}{\sigma}\right]
$$

is a reduced $\wp_{\Delta}$-ideal, then the set

$$
\left\{\frac{Q_{1}}{\sigma}, \frac{Q_{2}}{\sigma}, \ldots, \frac{Q_{\ell}}{\sigma}\right\}
$$

represents the norms of all reduced ideals equivalent to $I$. This is achieved via the simple continued fraction expansion of $\alpha=(P+\sqrt{D}) / Q$.

Two important results needed in the next section are given as follows.

THEOREM 2.5. Suppose that $\Delta \equiv 1(\bmod 4)$ is a discriminant and $\ell\left(\omega_{\Delta}\right)=\ell$ with $Q_{j}$ defined for the simple continued fraction expansion of $\omega_{\Delta}$ as in (2.10). Then $Q_{j} \mid 2 D$ with $0<j<\ell$ if and only if $j=\ell / 2$.

Proof. See [5, Theorem 6.1.4, page 193].

Theorem 2.5 also has relevance to the solution of quadratic Diophantine equations (see [9]).

THEOREM 2.6. If $\Delta>0$ is a discriminant, $\varepsilon_{\Delta}$ is the fundamental unit of $\odot_{\Delta}$, and $\ell=\ell\left(\omega_{\Delta}\right)$, then

$$
N\left(\varepsilon_{\Delta}\right)=(-1)^{\ell}
$$

Proof. See [5, Theorem 2.1.3, pages 51-52].

3. Class number two results. In this section, we use the following notation for our canonical polynomial:

$$
F_{\Delta}(x)=-x^{2}+(\sigma-1) x+\frac{D-\sigma+1}{\sigma^{2}},
$$

which is the real quadratic field analogue of the Euler-Rabinowitsch polynomial $x^{2}+$ $x+(1-D) / 4$ (see [5, Chapter 4 , pages 105-128]). The following is a general class number 2 criterion in terms of $F_{\Delta}(x)$.

THEOREM 3.1. Let $\Delta=D$ be a discriminant. If $q<\sqrt{D}$ is a prime properly dividing $D$ and $N\left(\varepsilon_{\Delta}\right)=-1$, then the following are equivalent:

(a) $h_{\Delta}=2$.

(b) Whenever $p \mid F_{\Delta}(x)$ for any prime $p<\sqrt{\Delta} / 2$ and $1 \leq x \leq \sqrt{\Delta} / 2$, then $p$ is some $Q_{j} / 2$ for some $j$ with $1 \leq j \leq \ell(\alpha)=\ell$, where either $\alpha=\omega_{\Delta}$ or $\alpha=\left(b_{q}+\omega_{\Delta}\right) / q$, where the ideal $2=\left[q, b_{q}+\omega_{\Delta}\right]$ is the $\wp_{\Delta}$-prime above $q$.

Proof. We observe that $N\left(\varepsilon_{\Delta}\right)=-1$ guarantees that $\ell\left(\omega_{\Delta}\right)$ is odd by Theorem 2.6. If $2=\left[q, b_{q}+\omega_{\Delta}\right]$ is principal, then by Theorem $2.5, q=Q_{\ell / 2} / 2$, so $\ell$ is even, contradicting the initial statement. Moreover, $2^{2} \sim 1$ since 2 is ramified in $\odot_{\Delta}$ (see 
[8, Exercise 4.7(c), page 20]). We have shown that $2 \nsim 1$ and $2^{2} \sim 1$. Thus, we have the principal class and the class of order 2 containing 2 in $\mathscr{b}_{\Delta}$. Now if (b) holds, we have to show that there are no others therein. By Theorem $2.3, \mathscr{C}_{\Delta}$ is generated by the non-inert prime ideals of norm $p<\sqrt{\Delta} / 2$. Thus, by (b), we need only to show that any prime $p<\sqrt{\Delta} / 2$ must divide $F_{\Delta}(x)$ for some positive $x<\sqrt{\Delta} / 2$, since in that case $p=Q_{j} / \sigma$ in one of the aforementioned two ideal classes, forcing an ideal $\mathscr{P}$ above $p$ to be either principal or equivalent to 2 , namely $h_{\Delta}=2$. Since we may write $\mathscr{P}=\left[p,-b+\omega_{\Delta}\right]$ where $0<b \leq p$, then $p \mid-N\left(\omega_{\Delta}-b\right)=F_{\Delta}(b)$. We have shown that (b) implies (a).

If (a) holds, then all ideals are either principal or equivalent to 2. Let $p<\sqrt{\Delta} / 2$ be any prime dividing $F_{\Delta}(x)$ for some positive $x<\sqrt{\Delta} / 2$. Therefore, $D \equiv(2 x-1)^{2}(\bmod p)$ so $p$ is not inert in $\oplus_{\Delta}$. Thus, since any $\oplus_{\Delta}$-ideal $\mathscr{P}$ above $p$ must be reduced, then it is either principal or equivalent to 2 , and $p=Q_{j} / 2$ for some positive $j \leq \ell$ in either the continued fraction expansion of $\omega_{\Delta}$ or $\left(b+\omega_{\Delta}\right) / q$ by Theorem 2.4.

EXAMPLE 3.2. From [11] (where there are a wealth of examples from which to choose), we select $D=29957$ for which $\ell\left(\omega_{\Delta}\right)=9$ and $h_{\Delta}=2$ so $N\left(\varepsilon_{\Delta}\right)=-1$ by Theorem 2.6. The prime divisors $p$ of $F_{\Delta}(x)=-x^{2}+x+7489$ with $p<\sqrt{D} / 2$ that appear as $Q_{j}$ in the principal class are $p=7=Q_{1} / 2$ and $p=83=Q_{4} / 2$. Since $D=29957=29 \cdot 1033$, then we may choose $q=29$ and $2=\left[29,14+\omega_{\Delta}\right]$. Also, in the simple continued fraction expansion of $\alpha=\left(14+\omega_{\Delta}\right) / 29$ (for which $\ell(\alpha)=15$ ), we achieve all of the other prime divisors $q<\sqrt{D} / 2$ of $F_{\Delta}(x)$ with $1 \leq x \leq 86$. Specifically, $Q_{0} / 2=29, Q_{2} / 2=11, Q_{3} / 2=47, Q_{5} / 2=73$, and $Q_{6} / 2=79$. This illustrates the power of Theorem 3.1.

The next result gives criteria for ERD types, which extends the work in [3, 4].

THEOREM 3.3. Let $\Delta$ be a discriminant with radicand $D \neq \equiv(\bmod 8)$ of ERD-type, and assume that $D \neq m^{2} \pm 2$ for any positive integer $m$. Then $2=\left[2, b+\omega_{\Delta}\right] \nsim 1$, and the following are equivalent:

(a) $h_{\Delta}=2$.

(b) Whenever there exists a positive integer $x \leq \sqrt{\Delta} / 2$ such that $p \mid F_{\Delta}(x)$ for any prime $p<\sqrt{\Delta} / 2$, then $p=Q_{j} / \sigma$ for some $j$ with $1 \leq j \leq \ell(\alpha)=\ell$, where either $\alpha=\omega_{\Delta}$ or $\alpha=\left(b_{2}+\omega_{\Delta}\right) / 2$, where the ideal $2=\left[2, b_{2}+\omega_{\Delta}\right]$ is the $\oplus_{\Delta}$-prime above 2 .

Lastly, if $D \equiv 1(\bmod 8)$, then $h_{\Delta}=2$ if and only if $D=65$ or 105 .

Proof. To see that $2 \times 1$, we invoke Theorem 2.4 and perform an exhaustive check of the $Q_{j} / \sigma$ in the simple continued fraction expansion of $\omega_{\Delta}$ for each ERD-type. Such tables may be found in [5, Theorem 3.2.1, pages 78-80], so we do not reproduce them here, and the result is easily verified therefrom.

Now assume that $h_{\Delta}=2$. Suppose that there exists a positive integer $x<\sqrt{\Delta} / 2$ and a prime $p$ such that $p \mid F_{\Delta}(x)$ where $p<\sqrt{\Delta} / 2$. If $\mathscr{P}$ is an $\oplus_{\Delta}$-prime above $p$, then $N(\mathscr{P})=p$ given that $p$ is not inert since $D \equiv(\sigma x-\sigma+1)^{2}(\bmod p)$. That (b) holds, now follows from Theorem 2.4.

If (b) holds, then we have the class of $2 \nsim 1$ and the principal class. Showing that there are no others is done in exactly the same fashion as in the proof of Theorem 3.1.

The last statement on $D \equiv 1(\bmod 8)$ is [4, Theorem 3.2, page 92]. 
The following result addresses the case $D=m^{2} \pm 2$ which was excluded from Theorem 3.3.

THEOREM 3.4. Let $\Delta$ be a discriminant with associated radicand $D=m^{2} \pm 2(m \in \mathbb{Z})$, and let $q<\sqrt{D}$ be an odd prime dividing $D$. Then the following are equivalent:

(a) $h_{\Delta}=2$.

(b) Whenever $p \mid F_{\Delta}(x)$ for any prime $p<\sqrt{\Delta} / 2$ and $1 \leq x \leq \sqrt{\Delta} / 2$, then $p=Q_{j}$ for some positive integer $j \leq \ell(\alpha)=\ell$, where either $\alpha=\omega_{\Delta}$ or $\alpha=\left(b_{q}+\omega_{\Delta}\right) / q$, where the ideal $2=\left[q, b_{q}+\omega_{\Delta}\right]$ is the $\Theta_{\Delta}$-prime above $q$.

Proof. If $2 \sim 1$, then the fact that $q<\sqrt{D}$ allows us to use Theorem 2.4 to analyze the tables in [5, Theorem 3.2.1, pages 78-80] and conclude that either $q=2$ or $q=$ $2 m-3$ with $D=m^{2}-2$. Therefore,

$$
D=m^{2}-2=\left(\frac{q+3}{2}\right)^{2}-2,
$$

which means that there is an integer $t$ such that

$$
4 q t=q^{2}+6 q+1
$$

which makes a contradiction since this forces $q \mid 1$. Thus, $2 \times 1$. However, since 2 is ramified, then $2^{2} \sim 1$, so we have the principal class and the class of 2 . The proof now proceeds as in the proofs of Theorems 3.1 and 3.3.

REMARK 3.5. The assumption in the hypothesis of Theorem 3.3, that there exists a prime $q<\sqrt{D}$, guarantees that there does not exist an ambiguous class without ambiguous ideas in it, since the assumption excludes those radicands $D=2 p$ where $p \equiv 1 \bmod 4$ such as $D=482$.

EXAMPLE 3.6. The radicand $D=58$ is of non-ERD type with $h_{\Delta}=2$ and $N\left(\varepsilon_{\Delta}\right)=-1$. In the simple continued fraction expansion of $\sqrt{58}$, the only $Q_{j} s$ are $1,6,7,9$, whereas in the simple continued fraction expansion of $\sqrt{58} / 2$, the only $Q_{j} s$ are $2,3,11$. Observe that the prime divisors 2 and 3 of $F_{\Delta}(x)=-x^{2}+58$ for $1 \leq x \leq \sqrt{58}$ appear in the simple continued fraction expansion of $\sqrt{58} / 2$, whereas the only prime divisor 7 of $F_{\Delta}(x)$ in that range appears in the principal class.

EXAMPLE 3.7. If $D=1298=2 \cdot 11 \cdot 59=36^{2}+2$, then $q=11$ and $h_{\Delta}=2$. In the simple continued fraction expansion of $\sqrt{1298}$ the only $Q_{j} s$ are 1 and 2, whereas in the simple continued fraction expansion of $\sqrt{1298} / 11$, the only $Q_{j} s$ are $11,19,22,29$, 37 , 38. This illustrates Theorem 3.4 since we observe that the only odd primes $p<$ $\sqrt{D}=\sqrt{\Delta} / 2$ dividing $F_{\Delta}(x)=-x^{2}+1298$ with $1 \leq x \leq \sqrt{D}$ are $p=11$ at $x=11,22,33$; $p=19$ at $x=5,14,24,33$; and $p=29$ at $x=14,15$.

REMARK 3.8. Theorem 3.4 only misses the case $D=2 q$ of the form $D=m^{2} \pm 2$ since the forms for $D$ to take when $h_{\Delta}=2$ are well known (see [8, Theorem 3.70, page 162]). Moreover, it can be shown that if $D=m^{2} \pm 2$ and the least odd prime quadratic residue exceeds $\sqrt{D}$, then $h_{\Delta}=1$ (see [5]).

Based upon the above and [10, Theorem 3.2, page 99] we pose the following. 
CONJECTURE 3.9. If $D=m^{2} \pm 2$ is squarefree, then $h_{\Delta}=2$ if and only if

$$
\begin{aligned}
& D \in\{34,66,102,119,123,146,194,258,287, \\
&402,482,527,623,678,782,843,902,1022,1298\} .
\end{aligned}
$$

REMARK 3.10. Using the techniques outlined in [5, pages 172-186], it can be shown that Conjecture 3.9 holds with one possible exceptional value, the existence of which would be a counterexample to the generalized Riemann hypothesis.

Recently in [1], Andrew Granville, the author, and Hugh Williams were able to unconditionally verify a conjecture made at the end of Chapter six of [5], which had theretofore only been known to hold under the conditions mentioned in Remark 3.10. It is, however, unlikely that those techniques can be used to unconditionally verify Conjecture 3.9.

ACKNOWLEDGEMENTS. The author's research is supported by Natural Sciences and Engineering Research Council (NSERC) Canada grant \# A8484. Also, the author welcomes the opportunity to thank the four (anonymous) referees for their comments, which helped to clarify presentation and minimize typos.

\section{REFERENCES}

[1] A. Granville, R. A. Mollin, and H. C. Williams, An upper bound on the least inert prime in a real quadratic field, Canad. J. Math. 52 (2000), no. 2, 369-380. MR 2001d:11123.

[2] M. D. Hendy, Prime quadratics associated with complex quadratic fields of class number two, Proc. Amer. Math. Soc. 43 (1974), 253-260. MR 49\#2648. Zbl 255.12001.

[3] S. Louboutin, R. A. Mollin, and H. C. Williams, Class groups of exponent two in real quadratic fields, Advances in Number Theory (Kingston, 1991), Oxford University Press, New York, 1993, pp. 499-513. MR 96j:11153. Zbl 795.11057.

[4] R. A. Mollin, Applications of a new class number two criterion for real quadratic fields, Computational Number Theory (Debrecen, 1989), de Gruyter, Berlin, 1991, pp. 8394. MR 93e:11133. Zbl 734.11057.

[5] _ Quadratics, CRC Press Series on Discrete Mathematics and its Application, CRC Press, Florida, 1996. MR 97e:11135. Zbl 858.11001.

[6] _ Prime-producing quadratics, Amer. Math. Monthly 104 (1997), no. 6, 529-544. MR 98h:11113. Zbl 886.11053.

[7] _ Fundamental Number Theory with Applications, CRC Press Series on Discrete Mathematics and its Applications, CRC Press, Florida, 1998. Zbl 943.11001.

[8] _ Algebraic Number Theory, CRC Press Series on Discrete Mathematics and its Applications, Chapman \& Hall/CRC, Florida, 1999. MR 2000e:11130. Zbl 930.11001.

[9] R. A. Mollin and A. J. van der Poorten, Continued fractions, Jacobi symbols, and quadratic Diophantine equations, Canad. Math. Bull. 43 (2000), no. 2, 218-225. MR 2001a:11049.

[10] R. A. Mollin and H. C. Williams, On a solution of a class number two problem for a family of real quadratic fields, Computational Number Theory (Debrecen 1989), de Gruyter, Berlin, 1991, pp. 95-101. MR 93d:11118. Zbl 734.11058.

[11]_. On real quadratic fields of class number two, Math. Comp. 59 (1992), no. 200, 625-632. MR 93a:11089. Zbl 774.11061.

Richard A. Mollin: Mathematics Department, University of Calgary, Calgary, aB, CANADA T2N 1N4

E-mail address: ramo11 inamath.uca1gary.ca

URL: http://www. math.ucalgary.ca/ ramol1 in/ 


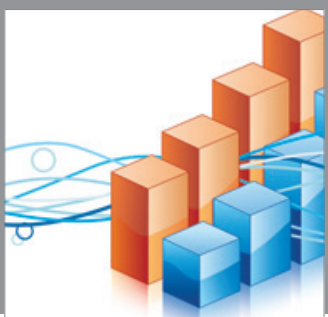

Advances in

Operations Research

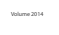

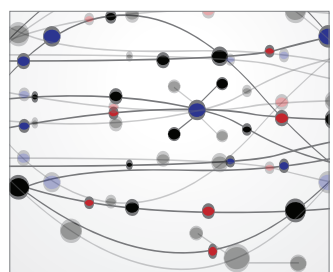

\section{The Scientific} World Journal
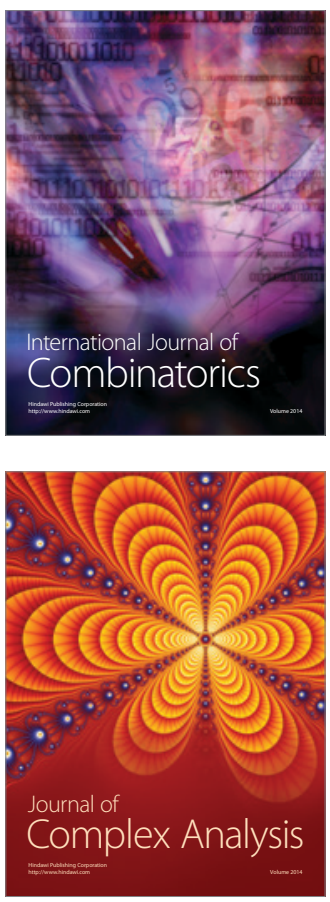

International Journal of

Mathematics and

Mathematical

Sciences
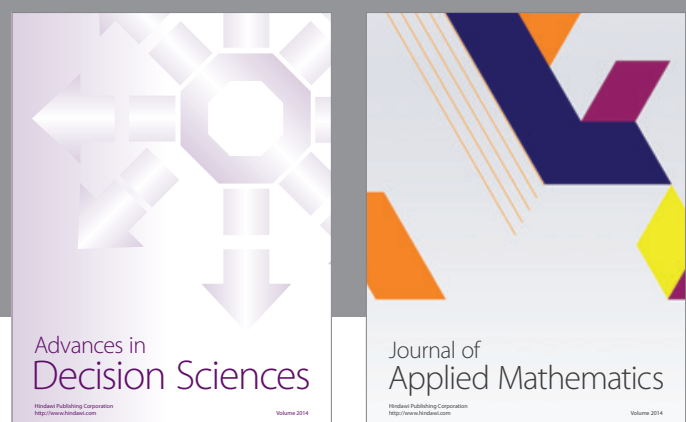

Journal of

Applied Mathematics
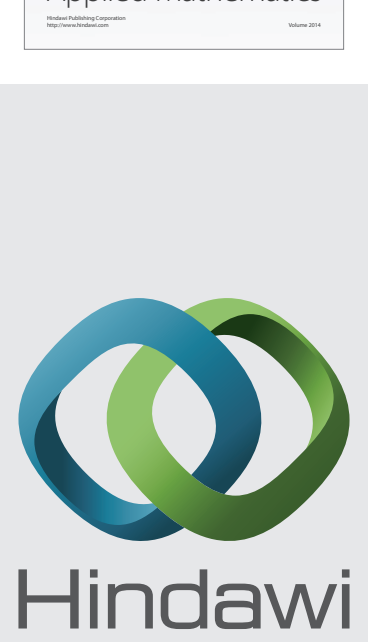

Submit your manuscripts at http://www.hindawi.com
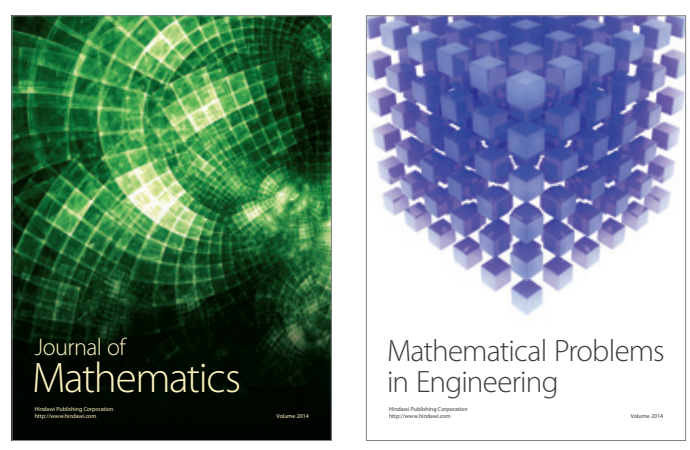

Mathematical Problems in Engineering
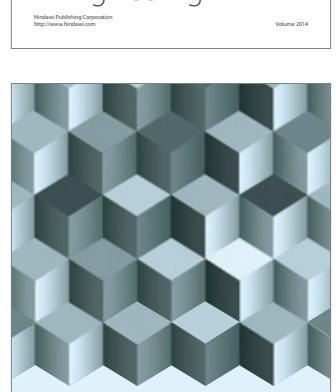

Journal of

Function Spaces
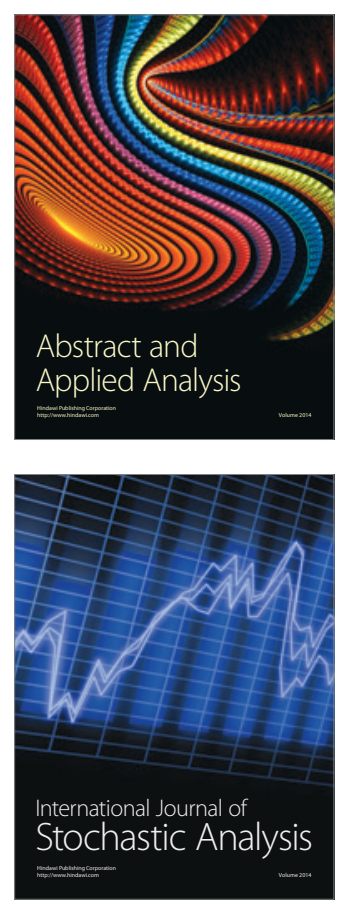

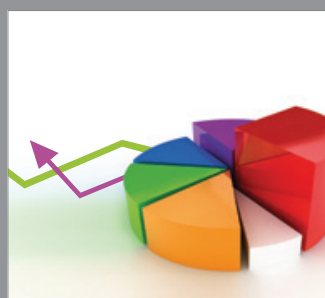

ournal of

Probability and Statistics

Promensencen
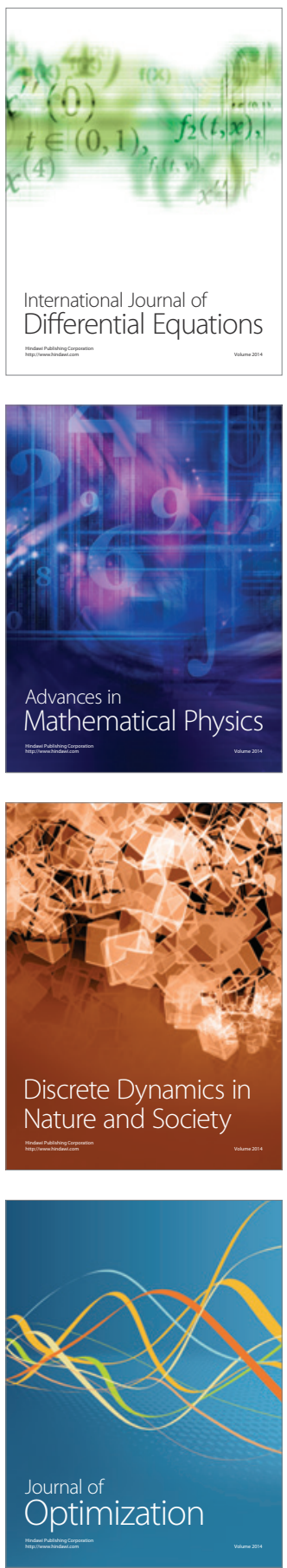